\title{
Hajj and Umra smart mobile Application: Culture and Usability Study
}

\author{
Firas Omar \\ Department of Software Engineering, Zarqa University, Jordan \\ fomar@zu.edu.jo \\ Tareq Alhmiedat \\ Department of Computer Science, Zarqa University, Jordan \\ t.alhmiedat@zu.edu.jo \\ Mohammad Hassouna \\ School of Computing and Mathematical Science \\ University of Greenwich, UK \\ M.Hassouna@greenwich.ac.uk
}

\begin{abstract}
Mobile technology is becoming more popular around the world. The importance of such technology relates to its capability of allowing the user of performing many different daily basis tasks. Despite the progress made in the mobile application field, there are still some boundaries and limitations in using it. Some of these difficulties are connected directly to the Culture. Other difficulties are related to the experience in using such technology. This research aims to find out the main restrictions and obstacles which limit the use of mobile handsets as an Islamic smart Hajj and Umra Application. The research aim extends to studying the effect of cultural issues on people's use of the internet on a mobile phone. The research reported here is based on participants from the Hashemite Kingdome of Jordan.
\end{abstract}

\section{Indexing terms/Keywords}

Culture, $\mathrm{HCl}$, Usability.

\section{Academic Discipline And Sub-Disciplines}

This paper addresses several important aspects such as, Culture, age, mobile human cumpoter interaction, trust,familiarity and usability. These aspect tends to answer the problem arose for this paper. The aspects also tends to solve the issues assiosiated with the topic of the paper through the understanding of each of them and addressing each aspect thoroughly within the steps of composing this work.

\section{SUBJECT CLASSIFICATION}

Computer Sciences and software engineering.

\section{TYPE (METHOD/APPROACH)}

To achieve the broad aim of this research, it was important to conduct an initial study to clarify the problem. Therefore, the first step of this research was to develop and validate a questionnaire to gain information on the problem area.

The next stage is to try to address the issues generated from the results obtained from the questionnaire. This will highlight the main obstacles and barriers of using smart mobile phones in Hajj and Umra. Participants will be randomly from thoese who had performed Hajj or Umra. The minimum number of participants should not be less than 30; no upper limit is required, however. The lower limit is more important because it allows the use of the central limit theorem and the normality of data assumption at the statistical analysis stage.

The experiment will be divided into three parts. In the first part, participants will be asked to fill in a questionnaire designed to understand the main problems that the proposed system will try to solve. The second part of the study will involve designing and developing the system which will be specially developed to work under a smart phone environment. Finally, participants will be asked to complete a usability questionnaire at the end of the experiment. Participants will be observed while completing each task of the experiment.

\section{Council for Innovative Research}

Peer Review Research Publishing System

Journal: INTERNATIONAL JOURNAL OF COMPUTERS \& TECHNOLOGY

Vol 10, No 3

editor@cirworld.com

www.cirworld.com, member.cirworld.com 


\section{INTRODUCTION}

Day by day, the need to computerize and simplify our life comes to take place in our life. As a Muslim person, the need to guide and advise the Muslim during the journey to the sacred places in both Makkah and Madena could be very beneficial and consequently will make His/hers journey to perform Hajj or Umra easier and more enjoyable. To insure such beneficial outcome, the design and implementation of this smart mobile application has taken so many considerations into account. Issues such as trust, culture and usability were considered through the process of design and implementation of the application.

\section{Literature}

\subsection{Culture}

According to Hofstede (2001), culture can be defined as "the collective programming of the mind which distinguishes the members of one group from people from another" (Hofstede,2001. P.4). Additionally, culture cannot be clearly understood by studying a single person or individual; it

can only be dealt with as a set of shared characteristics within a group of people that affect the behaviors of individual members by providing rules for that group.

Additionally, culture combines itself on both the visible aspects of a group and a wide range of elusive aspects, including values, thoughts and behavior. Culture can be conceptualized as a set of major variables that informs the responses of individuals in that culture to new practices, ideas

and technologies, such as mobile data services.

One of the earliest definitions of culture was given by Tylor (1871). He described culture as "That complex whole which includes knowledge, belief, art, morals, law, customs and any other capabilities and habits acquired by man"(Tylor,1871. P.410). Harris (1987) stated that

cultures can possibly be learned or acquired, and they reflect patterns of thinking, feeling and acting. One of the most important definitions of culture was given by Kluckhohn (1951), who declared that "Culture consists of patterned ways of thinking, feeling and reacting acquired and transmitted mainly by symbols consisting of the distinctive achievements of human groups including their embodiments in artifacts, the essential core of culture consists of traditional and historical derived and selected ideas and especially their attached values" (Kluckhohn, 1951, P.86). Hofstede highlighted "Values" as the building bricks of any culture which are set early

in childhood and shapes subjective definition of rationality. Hofstede added that values have both strength and direction, or alternatively they have a size and a sign.

Culture can be defined as a way of life, how people in a specific area or society learn how to dress, eat and communicate together. Particularly, it refers to socially learned and absorbed behaviors, beliefs and values that member of the same group or society share with others. It is absolutely a fact that different cultures exist in the world, each of which has its own cultural aspects and features, such as symbols and language, rules and values.

Culture can be viewed as the number of attributes that people acquire from their childhood rearing. These attributes are fundamentally associated with their surroundings and environment that influence the responses of people in that culture to precede with the production of new ideas, use of new technology and practice it, takinginto consideration that culture may affect

the way people act and behave in general.

\subsection{Age}

\subsubsection{OLD}

The percentage of older adults in developing countries is growing up day by day and their mobile interaction has increased because of better general health and financial resources (Goodman and Gray 2003). However, as people age, they experience a normal decline in a number of abilities, such as sensory information and mental abilities. Therefore, to remain liberated older adults often require more support. As the percentage of older adult's increases, the possibility of relying on human care contributors to provide this support declines, increasing the need for other types of support such as mobile computer devices (Goodman et al. 2004).

Mobile devices can support older adults in lots of forms; for example mobile phones can help older adults remain connected, new memory aids that enable them to remember important information (Inglis et al. 2003), and transportable game systems that provides them with fun and interesting mental exercises(Nintendo 3DS).

Older adults, though, have been slower to adopt mobile computer technologies. In addition, they found such technologies pretty difficult to be used (Kurniawan et al. 2006). For instance, Ofcom survey conducted at the UK in 2006 , only $49 \%$ of seniors (age $65+$ ), compared with $82 \%$ of all adults participants had reported owning a mobile phone (Leung et al. 2009). From these seniors, 
$44 \%$ reported that they can listen to voicemail messages and $29 \%$ reported that they can send a text message (compared with $83 \%$ and $81 \%$ of all adults, individually). Recent $\mathrm{HCl}$ research has tested many different usability issues that may in its turn explain the unwillingness of older adults to adopt mobile devices e.g. sigh problems (Jacko et al. 2002) hard to act with interface (Ziefle and Bay 2005).

\subsubsection{YOUNG:}

Younger users are an important consumer section as they represent a group of almost 70 million users in the USA with a major spending power (Kumar, A \& Lim, H, 2006). Younger users typically spend about $\$ 187$ billion annually or $\$ 260$ per person monthly in most of the industrial sections including wireless communication .Moreover, Younger users are important for market experts because of the influence that they apply on their families in the form of purchase decisions (Renn and Arnold, 2003).

Younger users are frequently and usually the early adopters of any new technologies and are

broad users of the internet. Likewise internet, Younger users are large users of mobile services. In relation of mobile service usage, the latest study reports that more than 50 Percent of US consumers in the age group of 15-24 possess a mobile phone and these users number is more than all other users in terms of number of calls placed, minutes consumed, wireless data transmitted/received and messages sent/received (Wilson,2003).

Short message services (SMS) amounts are rising frequently among Younger users. A recent study specifies that majority of Younger users communication takes the form of sending and receiving SMS with an average of 126 messages sent each month (Kumar, A \& Lim, H, 2006) (Perez and Gen, 2006).

Mobile phones are often known as a tool of self-expression and independence for the younger generations (Ling, 2001; Taylor and Harper, 2001). For instance, Younger users often do personalize their phones by screensavers and message tones, downloading exclusive ring tones even if it was expensive. In addition, Younger users use mobile phones as an important method to uphold peer relations. As Younger users appear to be in the key stream of data service users in the USA, and as mobile data services are a main source of income for mobile carriers, Younger users are a significant element for mobile carriers markets (Kumar, A \& Lim, H, 2006).

\subsection{Mobile Human-Computer Interaction}

The ACM defines human-computer interaction $(\mathrm{HCl})$ as "a discipline concerned with the design, evaluation and implementation of interactive computing systems for human use and with the study of major phenomena surrounding them" (Hewett et al., 1992). This definition positions

mobile $\mathrm{HCl}$ as a sub-area focusing on one species of interactive computing systems-the portable and handheld computer. The significant aspect of mobile $\mathrm{HCl}$ is user mobility. The user's agency for physical movement changes the conditions of interaction so profoundly that mobility is one of the key challenges for post-millennium research.

Mobile prototypes are being developed in special application domains that tend not to refer to work done in the core of mobile HCl. The lack of valid and actionable results, methods, and theories may be part of the reason why substantial investments in mobile technologies can fail. The case of wireless application protocol (WAP) in Europe is a good example from the recent past. Academic research should not be a slave to the development of technology, but it should steer efforts across application domains.

Nonetheless, the first 10 years of mobile $\mathrm{HCl}$ research cannot be judged as being a failure. Mobile $\mathrm{HCl}$ has been, by and large, a technology-focused enterprise (Kjeldskov and Graham,

2003; York and Pendharkar, 2004) and a model of fruitful industry-academia relationships. Numerous commercial products and services have been anticipated by academic publications and almost all major manufacturers and network operators have active research divisions that take

part in academic conferences.

Location-based services, awareness, remote monitoring, media-capturing and sharing, browsers, online banking, business processes, games, office documents, voice communications, short messaging, email, and input interfaces are prime examples of research areas that have contributed to industry efforts. However, the bulk of this work has been local in a sense, specific to the application or setting, without generalizable understanding that carries over to new applications and settings. We need stronger basic science without letting industry needs "off the hook."

The current research in this general area is breaking new ground by bringing into focus the expanding scope of mobile $\mathrm{HCl}$, from the user interfaces designed for the moving user to the immediate situations at hand, all the way to more advanced social and organizational cultures. In the end, the core of mobile $\mathrm{HCl}$ that links these scopes is how the users' actions will change their relation to their contexts, and how this in turn affects the use of computers. In other words, the unique aspect of mobile $\mathrm{HCl}$ is the strong influence of the environment, almost to the extent we could talk about human-environment-computer interaction (Kjeldskov and Graham, 2003; York and Pendharkar, 2004).

Mobility is essentially about change, something transforming to something else as a consequence of a user's cycle of actions that provide new resources and place constraints for the resulting actions. Mobility is therefore both a limiting as an enabling factor. 
Herein liesthe greatest advantage of mobile technologies over stationary tools as a platform for $\mathrm{HCl}$. They should not simply be conceived of as smaller or lower versions of desktop applications; they enable a host of new services that leverage their contexts for the benefit of the user. The environment is not only a condition for achieving something, but it can be actively exploited. The full spectrum of the conditions that are relevant spans the physiology of joint movement as well as to the sociology of human mobility.

Mobile $\mathrm{HCl}$ has been an active area for more than a decade, yet this research will be targeting not all of mobile $\mathrm{HCl}$, but specifically the problems of user mobility and mobile use. The activities studied in $\mathrm{HCl}$ literature are illustrative of how mobile devices are becoming one of the most pervasive platforms for $\mathrm{HCl}$. The diversity of frameworks needed to address mobility in these papers implies that we do not have a unifying framework for mobility, and may not have in the future. Instead, the phenomenon is tackled from multiple perspectives that are irreducible to each other.

The fluid and natural interactions enabled by modelling physics are common in games and are moving into mobile phones. Physics engines have been used in games for many years to make movements and interactions more realistic, or alternatively to be able to play with the laws to physics to create new game play. Mobile phones now have the processing power to do some of the same things in their interactions. Signs such as flicking pages to turn them or throwing a scrollbar and seeing it slow down the further it scrolls are easy for users to understand and make for playful and engaging interactions. Many phones now also join accelerometers so that the in the future the whole device could be used to gesture, for example tilting to zoom or scroll. Eslambolchilar and Murray-Smith (2008) suggest that techniques such as zooming and scrolling based on dynamic models for mobile phones are in their infancy, with many techniques hard-coded into applications, rather than using general models that could be applied across the whole

interface. One reason for this is that many interaction designers are not familiar with the concepts, specification mechanisms and calibration tools needed to make these techniques work. Eslambolchilar and Murray-Smith (2008) proposed a theoretical framework to underpin these dynamic interactions and show how it can be used to model tiltbased speed-dependent automatic zooming.

Until recently, most mobile devices used a small keyboard, or a touchscreen and stylus for input. Things are now changing with a new generation of devices based on touchscreens operated by fingers. The removal of the physical keyboard allows designers more flexibility to alter the size and shape of a device and to allow input on different parts of it, for example on the back, so that the screen is not obscured during input. However, it is not clear how effective users will be at interacting on devices such as these. It requires them to use fingers, thumbs or two hands together in different ways, sometimes on the front of the device and sometimes on the back. Wobbrock, Myers and Aung (2008) presented three detailed studies characterising input using different hand positions on the front and back of the mobile phone devices to show the performance of these different surfaces. Their results show that the index finger is good for input on the front or back surface of the mobile device, while the thumb is usually preferred to be used on the back rather than front. However, the index finger has its limitations when complex motions are needed, wherein it performs better on the front of the device. These studies can help device designers understand how to create more effective interactions in future devices which may have very different form factors to the ones we are currently using.

Fickas, Sohlberg and Hung (2008) pointed that there has been a very fast growth in the use of in- car navigation devices over the past few years. The same is starting to happen in the area of pedestrian navigation, with many new mobile phones including GPS around us, receivers and mapping software. This has unlimited possibilities for all pedestrians, but there may be certain

benefits for people with cognitive impairments. Cognitive impairments can affect navigation skills which are fundamental to personal independence, community integration and travel. The right technology could help overcome some of these problems. Due to their disability, cognitively impaired people's needs and requirements are different and standard navigation tools are not always suitable, particularly in the way that route following assistance is presented. Fickas, Sohlberg and Hung (2008) studied a range of different presentation methods to discover the most effective way to provide route-following assistance for this user group. They studied the use of aerial maps, point of view maps, and audio and textual directions with a group of twenty cognitively impaired users.

Results showed that speech-based directions were the most effective, followed by text reminders. Speech was also given the highest preference rating by the study participants. The main reason

for this is that it placed the lowest request on visual attention, a resource that was required in

order to navigate their way through. The results of this work may also be beneficial to designers of general pedestrian navigation tools as route following can be cognitively demanding for everyone, and reducing the load on visual attention will allow all users to focus on the environment they are moving through.

Trains, buses, planes and taxis ought to be prime contexts of use for mobile devices. Using

mobile devices while travelling is not only common; it is the selling point of business phones and PDAs. There is something in the "betweenness" of travelling that is easily seen as promising for mobile technologies. In their article entitled "Mobile technologies in mobile spaces: Findings from the context of train travel,"Axtell, Hislop and Whittaker (2008) investigated train travellers in the UK. Their argument was a strong empirical study revealing the physical, material, technological, social, and organizational problems of interaction while travelling. On the one hand, despite owning a good number of mobile devices, travellers are not able to do work

anytime or anywhere they want. At times they have to limit their work to those tasks where communication is not 
necessary, and they may be forced to return to pens and papers to do the job. On the other hand, workers are not powerless in face of resource deprivations, but they exhibit quick-witted local adaptations to overcome restraints; and they plan themselves to address these limitations within a specific timeframe; in best cases they turn these limitations to their benefit. The broader point that Axtell, Hislop, and Whittaker (2008) make is that users place not only to the restraints of their immediate surroundings; they adjust their practices to organizational expectations and norms. They conclude with a convincing critique of naive notions of "context"; even train travel is no clear-cut, unitary context of use, but users actively construct conditions for work by adapting local resources to their practices and their devices, yet they are continuously sensitive to non-present, non-visible demands.

In their article entitled "Organisational usability of mobile computing-volatility and control in mobile foreign-exchange trading", Sorensen and Al-Taitoon (2008) expanded on the point that non-visible conditions shape the use of mobile technology. They traced the long-term evolution of technologies of foreign exchange trading from face-to-face bartering in the Babylonian civilization to the modern 24-hour on-floor and off-premises model, whereby traders orchestrate their use of phones, pagers and PDAs. Al-Taitoon and Sorensen (2008) studiedthetraders of a Middle Eastern bank who used their mobile devices to make decisions in face of uncertainty and

shifting risk. On the one hand, market volatility creates a need for off-premises trading and quick decision making under uncertainty, but on the other, this clashes with the need for organizational control. Mobile traders need special technical and social skills to manage with this problem. Al- Taitoon and Sorensen (2008) presented evidence for the claim that what was understood as the usability of a mobile system depends critically on balancing individual and organizational control. Here, the strong role of mobile devices appears in the context of dynamically switching spheres of work and personal identities that demands looser coupling and more discretion.

Studies since the turn of the millennium (Kopomaa, 2000; Katz and Aakhus, 2002; Ling,

2004)have repeatedly pointed out the importance of mobile phones in the coordination of interpersonal activities and (at a higher level) in the management of one's social networks. For many years now, Japan has been located at the lead of mobile services. Recently, the country has witnessed a massive jump from the use of pagers and SMS to mobile email. In their article entitled "Kei-Tying teens: using mobile phone e-mail to bond, bridge, and break with social ties-a study of Japanese adolescents",Boase and Kobayashi (2008) reported that Japanese youths not only established relationships with phones; they use phones to actively add new contact points to their networks. Japanese teenagers use mobile e-mail to bond and bridge with

their friends, but not to break ties. These findings are exciting not only because they are telling of a recent expansion in the interpersonal uses of mobile phones, but also as they are telling of how mobile devices expand to the territory of social networking sites.

Boyd and Ellison (2008) defined social networking sites as web-based services that "allow individuals to construct a public or semi-public profile within a bounded system, clear a list of other users with whom they share a connection, and finally view and traverse their list of connections and those made by others within the system". The findings of Boase and Kobayashi (2008) bring about an attractive idea: that mobile e-mail, although not designed for this purpose, can be and is being used for social networking activities. Instead of traversing digital connections, phones are chosen by users to "digitalize" connections that are created face-to-face. The authors report that meetings in halls and lobbies that are too brief for face-to-face conversations are opportunities to exchange e-mail addresses, and connection is maintained by sending messages throughout the day. E-mail messages reach recipients across device boundaries and help maintain connections, no matter whether one is on or off the desktop. The authors also report an

association between the amount of bridging a user does and the strength of use: more bridging leads to more use of mobile phones. Mobile e-mail may be a sign of what may turn into Web 2.0 on mobile phones.

In their article "Theorizing mobility in community networks", Carroll and Rosson (2008)analyzed the possibility of mobile technologies supporting community networks. Their target community was a rural town of 80,000 people located in the Appalachian Mountains with the purpose of facilitating information dissemination, discussion and collective activity. Their study was an exercise in and an extension of scenario-based analysis to the space of mobile services. Scenario- based analysis (Carroll, 2000) has been one of the most powerful ideas in design-oriented $\mathrm{HCl}$ during thelastdecade.

The term "the conundrum of human-computer interaction" is explained by Carrol (2000) thus: an imaginative person can entertain almost any form of interaction or interface, yet there are hard limits on the use of technology. A designer can assume a user who can type with two hands while walking, and a marketing researcher that train travellers are interested in killing time by watching advertisements on their devices. Carroll and Rosson (2008) take the reader by the hand and lead them through a process to show how their framework lends itself to difficult phenomena like communities and mobility. The authors not only elaborate the notion of community for this purpose, but they draw from theoretical and empirical literature to build a scientific basis for their design choices. They developed principles for wireless community networks and discuss the special nature of mobility. The resulting scenarios show convincing ways of using the physical world and the proximity of community members. The article has a timely focus, since communities and more generally "social networking" is an important ingredient of the Web 2.0 movement.

As a starting point for their article entitled "Storied Spaces: Cultural accounts of mobility, technology, and environmental knowing", Brewer and Dourish (2008) referred to the sociologist John Urry (2000), who suggested that mobility rather than society may be the primary animating metaphor for the present century. Brewer and Dourish (2008) wrote that: "If mobility is culturally shaped, then we must think about mobile technologies not so much as devices that help solve 
problems, but as sites at which social and cultural categories are enacted." By claiming that space is also a social product, they go beyond the argument of Harrison and Dourish (1996), that what we call space can be understood from the perspective of geometry and experience. Technological representations of space are never devoid of perspective, but are always implicated in ways of movement and knowing the world. They argue that cultural logics feature in the collective understanding of space, and that technologies are not uncertain about conflicts between logics. Brewer and Dourish (2008) developed this argument further through numerous examples

whereby they re-interpreted context-aware services from new perspectives of cultural legibility, literacy and legitimacy.

\subsection{Importance of Trust:}

Beatty et al. (2011) defined trust in a broad sense as the confidence an individual has in his/her favourable expectations of what other people will act with or do, based on previous interactions in many cases. Although previous individual or group (of individuals) previous behaviour cannot always guaranteethat the subject will behave as expected, increased trust is simply the belief that another party will behave as the subject believes.

Through this trust, people reduce the complicity of understanding others into manageably understandable units, making an unjustifiable belief about the future subjectively justifiable (Dikomaet al., 2010; Flink and Schreiterer, 2010). Without trusting others in this way, people would be met with the incomprehensible complexity of considering every possible possibility

of every person around before deciding how to act. Such complexity would be so overpowering that, in many cases people would choose to stop doing a thing.

Trust is not the only a complexity reduction method; rules are also substantial techniques for reducing complexity. However, even with the existence of rules, trust is vital because there is no guarantee that other people will fully stand by them (Bachmann and Inkpen, 2011). Trust does not really enable people to control or even expect others behaviour without error, but it does make it possible for people to create an understandable organization of their interactions with others (Luhmann, 1988;FlinkandSchreiterer, 2010). Trust is a prerequisite of behaviour and is no less than a "basic fact of social life" (Luhmann,1979, p. 4).

However, the relative importance of trust depends on the nature and the complexity of interaction with other people. The greater the necessity to interact with other people and one's own exposure to their misbehaviour, the greater the need to trust (Rousseau et al., 1998;Salamon and Robinson,

2008; Flinkand Schreiterer, 2010). Trust is therefore intrinsically complex, multidimensional (Gillespie and Dietz, 2009), and therefore context-dependent (Flinkand Schreiterer, 2010). The early psychological and sociological studies on trust defined it as a belief that other people would achieve their expected favourable commitments (Flink andSchreiterer, 2010). Recent business research has taken a similar stand by defining trust as the expectation that other individuals or companies will behave morally dependably, and will fulfil their expected commitments under conditions of vulnerability and interdependence (Schoorman et al.,2007).

Unsurprisingly, trust has a significant effect on business relationships in general (SalamonandRobinson, 2008). It reduces the need for extensive negotiations, detail-resolution comprehensive legislation and enforced regulation, and fitted organizational control (Schoorman et al.,2007). Trust encourages long-term orientation (SalamonandRobinson, 2008), and increases the acceptance of interdependence and creates commitment (Cannon et al., 2010).

Trust when applied also reduces supposed risk (Cannon et al., 2010) can reduce transaction costs when warranted (SalamonandRobinson, 2008), and is to some extent important in almost any contractual agreement because of possible opportunistic behaviour of the other party. To conclude, trust determines the nature of the social and business order (SalamonandRobinson,

2008;Flink andSchreiterer, 2010) as well as the quality of business relationships. The observation that people need to trust in order to participate in an activity with another person and would rather abstain from any activity with others whom they do not trust (Luthmann, 1988)further supports these observations.

According to TomlisonandMayer (2009), trust in business "is the salient factor in determining the effectiveness of many relations". It is also considered to be a key promoter of behaviour in

general (Gefen, 2000). Its importance is not only in its role in defusing concerns of adaptable behaviour, but also because by resolving such concerns it reduces the need to invest in promised counter measures (Schoorman et al., 2007).

Similarly, lack of trust creates control-oriented and defensive communication that damages communication effectiveness and distorts crucial information (Schoop et al., 2010). In addition, it might result in an overall discouragement of the will to take risks(Flink and Schreiterer, 2010).

These effects of trust, especially the willingness to engage in activities where a person is unprotected to risk without the ability to control the related behaviour of others, and its

importance in successful acceptance of new technology (SalamonandRobinson, 2008), make trust a potentially important precondition for e-commerce, a fact about which internet and credit card industries are apparently well aware. 


\subsection{Trust in Mobility}

Over time, increased attention has been focused on the development process of trust in e-commerce. Trust became a serious topic for research because it is the cornerstone in creating satisfied and probable outcomes as a result of transactions(Gefenand Straub, 2003; Pavlou, 2003) Also, as explained by Salam, RaoandPegels(2003), trust can be developed by the trustee to positively create a trustor. Such perception is important to reduce the uncertainty and risks of transaction that exist for the individuals willing to trust about a potential or existing relationship- business, social or otherwise (Tomlisonand Mayer, 2009).

According to Mayer et al. (1995), trust is not taking risks as such, but rather it is an enthusiasm to take a risk. McKnight, Choudhury and Kacmar (2002) further defined trust in terms of ability, integrity and benevolence. Ability is related to skills and competencies of the trustee in a specific context. Integrity concerns if the trustee follows moral and ethical principles that are considered

to be acceptable by the trustor. Kindness concerns the degree to which the trustee has care or understanding towards the trustor. Perceptions of these elements will affect the trustor to have trust towards the trustee.

\subsection{Familiarity:}

Another way people subjectively lower uncertainty and simplify their relationships with others is familiarity. Familiarity is an understanding, often based on previous interactions, experiences,

and learning of what, why, where and when others do what they do (Beatty et al., 2011). By itself, familiarity and trust are distinctly different. Familiarity handles the understanding of the current actions of other people or of objects, while trust deals with opinions about the future actions of other people (FlinkandSchreiterer, 2010).

For example, familiarity with Amazon.com, one of the largest book selling internet vendors, would be the knowledge of how to search for books and information about them, and how to later order these books through the website interface. Familiarity in this context is a specific activity-based cognizance based on previous experience or learning of how to use the particular interface.

On the other hand, trust in Amazon.com, might involve providing credit card information based on the favourable belief (i.e. trust) that the information will not be wrongly used in any way in the future. Accordingly, familiarity and trust complement each other as complexity-reduction methods. Familiarity reduces uncertainty by establishing a solid reliable structure (FlinkandSchreiterer, 2010); trust reduces uncertainty by letting people hold "relatively reliable expectations" (Flink andSchreiterer, 2010) about other people's favourable future actions

(Flink andSchreiterer, 2010). In the case of using Amazon.com, familiarity in its turn will

reduce complexity through an understanding of how to search and buy books through the site and what the procedure involved.

Trust, on the other hand, would reduce other aspects of complexity by a priori ruling out unethical behaviour, such as misuse of credit card information. Trust and familiarity, however, are not of equal importance (Flink andSchreiterer, 2010), because trust relates to the unknown future actions of others, and these are inherently more dynamic, general, complex, risky and less specific. In the case of Amazon.com, users' trust should be more important when buying books, Mp3s etc. than when enquiring about books, not only because the consequences of credit card misuse deal with the future, but also because the nature of potential credit card misuse is more complex and risky.

Despite the fact that familiarity and trust are different, they correlate to each other. The reason for this is that trust in another person or organization is built when the other person or organization behaves in accordance with one's own favourable expectations of them. Since these favourablebehavioural expectations (trust) are naturally contextdependent, understanding the given context.

Familiarity is often an important antecedent (Flink and Schreiterer, 2010). Likewise, without familiarity with the context, it would be obvious that trust cannot be effectively attached to specific favourablebehaviours and thus cannot be as strongly conferred. Familiarity creates this background "precondition for trust" (Flink andSchreiterer, 2010.p.670). Once again in the case of Amazon.com, people's familiarity with the concept of secure internet communications could enable them to entertain specific beliefs concerning the security measures they expect from the vendor (this is trust). Likewise, buyers who are not aware of spying on the internet (lack of familiarity) have no reason to hold such expectations (trust).Another reason that familiarity can build trust is that familiarity not only provides an outline for future expectations, but also lets people create solid ideas of what to expect based on previous interactions (Cruz et al., 2010).

In many cases, prior experience is the basis of trust (Perrons, 2009) and familiarity can both create trust, when the experience was favourable, and ruin trust, if experience is not favourable (Flink and Schreiterer, 2010). In the case of Amazon.com, people familiar with Amazon.com had probably previously bought from the site and in the process had likely noticed that the vendor behaved in accordance with what they expected such as respect of privacy, accurate charges to their credit card account, and sending updates on the status of their orders.

\subsection{Usability:}


In the last few years, mobile devices reached a very popular place within the consumer lives that it became one of the very essentialgadgets. Mobile device functionality has expanded from just a device to press and dial numbers to the form of personal digital assistants (PDAs)(Barnum, 2002).

Currently, almost all mobile devices comprise a mobile phone, a calendar or memo, an alarm clock, an appointment schedule, a digital camera, an internet platform and many games. These functionalities were exclusively operating in PDAs before they became combined with mobile

phones, during which time it became increasingly important to learn how to evaluate their use and the optimum ways to design mobile devices' functionalities.

Usability, which is related directly to mobile services that run on mobile devices, must be concerned with the mobile user and outlines of what interfaces for mobile services are appreciated and expected by the user.

Most traditional usability studies focused on the user of stationary devices, who can control the situation (that is the environment) in which it is to be used. Running traditional testing for mobile devices increases the risk for unrelated results due to failing to consider mobile characteristics. Therefore, in order to produce effective end-user programming in mobile devices, it is essential to create a new usability testing method based on consideration of mobile devices' characteristics (Barnum, 2002).

Howarthet al. (2009) defined the term usability as the effectiveness, efficiency, and satisfaction in which users of a certain application is capable of achieving precise goals (ISO, 1998; Howarthet al., 2009). For many years, usability did not require justification in most quartersdue to continuously growing awareness of its value, and software production organizations'investment

in "doing usability", building privileged usability laboratories. This was achieved in many ways, such as buying usability equipment, conducting usability testing and training developers in usability engineering methods.

These investments have helped to make usability engineering an important part of the overall software development lifecycle. Accordingly, organizations want to maximize the effectiveness of their usability engineering processes. The literature, however, suggests that usability practitioners experience a number of difficulties that negatively impactthe effectiveness of their

work, which in turn impacts the effectiveness of the usability engineering processes within which they work.

According to Nielsen (1993), usability is defined as the measure of the quality the user practices when interacting with something like a traditional software application, web site, or any other device the user can operate in some way. Usability is not something that we can apply on a surface to give it extra shine at the last minute; it is deeply affected by every decision in design and development. In order to achieve additional usability and user satisfaction, we do not consider a single component but deem multiple components that are related to users and the product. Therefore, by focussingon the user, authentic usability can be characterized by the following (Nielsen, 1993):

1.Learnability: The system must be easy to master, so users can rapidly startcompleting work with the system.

2.Efficiency: The system must be easy to remember, so when the user has effectivelylearned the system, a high level of productivity will be achievable.

3.Memorability: The system must be easy to remember, so that the casual user will able capable of returning to the system after some period of not having used it, with no need to learn everything from the start point.

4.Errors: The system must have a low error rate, so that users will be making a smaller number of errors during the use of the system. Ifthey make errors, they can easily recover from them. In addition, terrible error possibilities must not happen.

5.Satisfaction: The system must be pleasing to use, so users are individually pleased during the time of usage.

In the case of a product, usability is decided by many factors such as the user's ease of use, user's perception of the quality of the product; the product's intuitiveness for the user; ease of learning and relearning, and the user's appreciation of the usefulness of the product (Barnum,2002).

In both users' and products' cases, usability must be planned by matching the use to a user, so that increasing the user's satisfaction of the product is the ultimate goal of applying usability. Therefore, useful usability in a computer system means that the application will provide the users with well-structured computing environments. According to Mayhew (1999), to achieve usability efficiently, a number of factors should be integrated:

1.Cognitive, perceptual, and motor capabilities and constraints of people in general.

2.Special and unique characteristics of the planned user population in particular.

3.Exclusive characteristics of the users' physical and social work environment.

4.Exclusive characteristics and requirements of the users' tasks, which will be supported by the product. 
5.Exclusive abilities and constraints of the selected software and or hardware and platform for the product.

Usability testing is the methodof doing usability evaluation on the product development (Lee and Grice, 2008). Generally, the goal of usability testing is to find asmany usability problems as possible during the test, afterwards, altering them before the product is released. Sometimes, the procedure for building usability testing ("usability engineering")startswith identifying a user, analysingtasks, and setting usability specifications (Leeand Grice, 2008).

It then passes throughdeveloping and testing prototypes and continues throughrepeated cycles of testing and development. Thus, the key goal of usability testing is to improve the usability of a product, and then, in the end, to increase the satisfaction of users.

\section{Questionnaire analysis}

Question 4: I face some problems while reading the supplications

Around \%39.5 of the sample agreed and strongly did for such question, they explained further

that carrying a book while performing Hajj or Umra is not comfortable and might keep them busy while performing such spiritual journey. It is worth to be mentioned that the majority of the participants who agreed and strongly did were from younger age group.

Others which were around \%47.4 of the sample refused such statement by selecting disagree and strongly disagree. They explained that it is not easy to brows the supplications from a mobile handset for them while performing Hajj or Umra. The process needs high concentration and using the mobile handset might make them astray from performing this worship. The added that they might be urged to answer a phone call or reply to a text message (SMS) which will in its turn will cause thoughts and ideas dispersion.

On the other hand, only \%13.2 of the sample has taken a neutral side of the question. The explained that they don't mind reading supplications from a smart phone or a handbook as long as it's going to perform what's required.

Question 5: When going to perform Hajj or Umra, I recall verses and supplications from my memory.

Nearly $42 \%$ of the sample agreed and strongly did for such question. Participants explained their answer and preference of such method by the fear of a technical issue with the mobile handset "What if the mobile battery runs out of power?" Most of the participants explained that it is much better top recall versus rather depending on the mobile handset. They explained further, smart phones battery life is very limited and any additional usage might end the user with a dead out of power piece of equipment. Besides that, their fear was justified by the theory of "Uncertainty Avoidance" by Hofstede (2001) and it is also justified by their culture which applies Uncertainty Avoidance in a direct way.

$13 \%$ of the sample strongly agreed on such statement. They explained that it is a must to try new things and it would potentially be a great chance to test such new technology and verify wither its going to work satisfactory for them or not.

$44 \%$ of the sample stood apart of the question and had chosen to be neutral. They explained that they are not sure if they going to benefit of using such technology for this sacred journey. This partially involves Uncertainty Avoidance as discussed earlier.

Question 6: The Times of "Tawaf" around the holy "Kaaba" and times of "Saeei" between Safa and Marwa make me busy of doing Duaa.

$63 \%$ of the sample agreed and strongly did for such question. The majority of the sample explained that when they are performing Hajj or Umra, it is one of the holy ceremonies to

perform "Tawaf" and it is a must to count each full round they are performing. As a consequence, it would be hard enough to perform such while reading and holding a smart phone. They also added some of the reasons that matches with the previous question, these reasons relates directly to Hofstede (2001) "Uncertainty Avoidance" Dimension. They explained that the smart phone might run out of battery and then they will face different sort of problems.

Over $23 \%$ of the sampledisagreed on such statement. They explained that they will follow up with each other to count how many rounds they had performed. In addition, they explained that some of the mobile handset applications could possibly have an application to count each round around the holy Kaaba.

Around $10 \%$ of the sample stood neutral, they had given no explanation for their choice.

Question 7: When going to perform Hajj or Umra I read verses and supplications from a handbook.

Around $58 \%$ of the sample agreed and strongly did. They explained their answer by explaining that reading from books is better than from the smart phone. This might be true in the case when the mobile handset is not completely charged or might run out of battery power very soon. It was also noticed that the majority of these who agreed were from the older group of participants. Older adults are usually slower to adopt mobile computer technologies. In addition, they found such technologies pretty difficult to be used (Kurniawan et al. 2006). This why it was observed that older people agreed with this statement

On the other hand, more than $23 \%$ of the sample disagreed and strongly did for this question. They explained that reading from the mobile handset might be easier for them. Besides that, it would be lighter to carry only the mobile handset rather than carrying a book to read the supplications from and other things beside the mobile phone. It was 
also noticed that younger users were keener on accepting such question rather than the older participants.

Only $18.4 \%$ of the sample were neutral, they didn't have any explanation for their response but

that they are confused between following the old method or starting to use the new technologies.

Question 8: Assuming that you went to Hajj or Umra with friends or family members, you don't

use your mobile phone as a communication method.

The majority of the sample (63\%) disagreed and strongly did for this question. They explained their answer by the fact that the Jordanian culture is a collectivism community and applies it in a direct way. collectivism is known for its well established and tight social boundaries and frameworks wherein people look out for their welfare and where personal goals comes in second place to those of the group (Choi et al., 2005). Jordanians tends to keep checking on each other continually, it is in their interest to assure that everyone in the group is in safe and no harm would threaten himlher. That's why they had disagreed on such question.

On the other hand, very few participants agreed on such question (8\%). They explained that it is highly important to isolate them self through this holy and sacred journey. They added, "It would be better to communicate when we are back to the hotel".

Around $29 \%$ of the sample were neutral, they explained that they are not really sure how would the mobile handset benefit them through the journey. They also added that it might be better to have a chat and check on each other after performing the "Hajj" or "Umra"

Question 9: Assuming that you went to Hajj or Umra with a friend or a relative, you would gather at a certain place to communicate with each other.

Once again, this question came to emphasis on the fact that Jordanians apply collectivism in their life in a direct way. Around $60 \%$ of the sample agreed and strongly agreed on such question, they explained that they must and need to check on each other in a repeated way. They also added that they won't feel comfortable not to check on each other and also get to know what activities they had performed through the day.

Only $8 \%$ of the sample disagreed on such statement. They had explained their answer with saying that "we are here a very short period of time, there is no time for friend and relatives". It might sound a bit aggressive but truly it is a short trip and won't last for more than a week so these participants preferred to invest in their time.

Around $34 \%$ of the sample kept them neutral. They explained their answer by that they don't know yet if they had the time to meet up with others or not. In addition, they added that they might not plan to meet anyone.

Question 11: I do care about the prayer time changes during travelling and I would really like to know about time changes.

The majority of the sample had chosen agree and strongly agree for this question. Around $87 \%$ of the sample were positive and supported such statement. They explained that the most important thing in the Muslim life is to keep up with the prayer time, it is highly important to investigate the prayer time changes in order to perform the 5 prayer within its correct time scope.

This high concern in prayer time proves that Jordanians apply the Action chaindimension by

Hall(1966). Action chains are defined as the sequences of events necessary to lead to the

achievement of a certain goal. Cultures vary in the degree to which members are committed to completing an action chain in a specific amount of time and in a certain order.

$\mathrm{n}$ addition, the Jordanian culture is considered to be a universalistic culture which was explained by Trompenaar (1993). This type of cultures Refers to the level in which an individual is committed to the set of standards and rules. It is obvious that the Jordanians are committed to perform prayers in a particular time. This was reflected by the acceptance of this statement of the questionnaire.

On the other hand, only 2 participants $(5 \%)$ disagreed on the statement. They explained that they can perform the prayers according to the time in their original city rather than following the changes in the holy places.

3 participants were neutral (8\%). They gave an explanation that states that they will perform the prayers with other pilgrims at its times.

Question 13: I care allot to enter al Haram al Makki from Al salam gate even thoug there are several other gates.

This question emphasis on the fact that Jordanian culture counts as Universalistic culture. According to Trompenaars (1993), this type of cultures Refers to the level in which an individual is committed to the set of standards and rules. It was advised by our prophet Mohammad (Peace be upon him) to follow his "Sunnah". "Sunnah" is defined by Sheikh Mohammad DeiaEIDinA'zamy "Sunnah, for Hadith scientists, refers to all that was narrated from the Prophet (PBUH), his acts, his sayings, and whatever he tacitly approved, in addition to all the reports which describe his physical attributes and character before the Mission or after it. There for, it was important to enter the "Haram Al Makki" from Alsalam gate. 
Only $13 \%$ of the sample had chosen disagree. They explained that they can enter al haram Al Makki from any gate. They also added that I might arrive from a different gate and it won't affect my hajj or Umra at all.

Neutrally, $21 \%$ of the sample had chosen neutral. The explained that the Al Salam gate might be crowded and it be difficult to enter from that particular gate. There are several other gates and we can enter Al haram from any one with not complications.

Question 15: I would really enjoy having such application installed on my mobile phone.

Over $92 \%$ of the sample had chosen Agree and strongly agree for this statement. They explained that the application is a new idea in Jordan and it is very easy to use. They added that this would aid the Muslim Pilgrims in a very positive way during the performance of Hajj and Umra.Participants mentioned that it was easy to interact with it in order to complete different tasks. Participants also added that the application would save lots of time because of avoiding

being lost and not following the correct sequence of worships in both Haram Makki and Haram Madani.On the other hand, only one participant (2.5\%) had chosen to disagree. No explanation was given. Two Participants (5\%) had chosen neutral. They explained that it would be better idea to test the

application while performing Hajj or Umra directly.

\section{Discussion:}

The results of this study address two aspects; mobile application design and culture influences.

For the application design, the term interaction design is defined as "The design of interactive products to support people in their everyday lives" (Preece et al., 2002). The process of designing the mobile application was the most important in this study.

According to Jones and Marsden (2006), the interaction design is distinguished in two ways. Firstly, in terms of the coverage of its vision: the design process is not only about technology or users who ultimately use designed applications; it is about building infrastructure that will improve the way the users are living and their life experience of that tool. Secondly, focus on real life aspects. That means that designers must beguided by an understanding of the impact of

design choices on the people who will end up using the application.

In this study, it was found that a massive share of the participants found the application easy to use; participants found it easy to interact with the application. It was easy and flexible to handle the application due to following Nielsen's (1994) design heuristics, such as: visibility of system status; match between systems and the real world; and flexibility and efficiency. Most of the answers explained that the application was very easy to use, which they found surprising; it was the first time they used applications that had real functionality.

It was also found that the application is useful and easily understandable. Participants in the study also added that the descriptions of the application fields are easy to read and clear to understand. Participants also reported that the application is very exact in which it can give full information, such as the location of the sacred places and what to perform there exactly. It was simple to use, with few fields and buttons, which made the users' interaction with the application more enjoyable.

The main focus in the process of the application development was on Nielsen's (1994) heuristic of match between system and the real world, which simplified the tasks in order to match the application function with the real world. Also, the Symbian UIQ (user interface platform for Symbian devices) guide explained by Jones and Marsden (2006) was taken into consideration through the design phases of the application.

The majority of the participants of the study found that the steps taken to complete the tasks were very easy, whichwas due to the successful deployment of Nielsen's (1994) visibility of system status heuristic. Participants in the study faced no difficulties at any stage in following the steps provided to them and found them very clear.

Participants in the study said that it had taken few steps to be guided throw the holy visit to perform hajj or Umra. The system found where the pilgrim were standing and what exactly to perform as worshiping or sacred places visit due the enabling GPS location system as a major feature of the application.

In addition, the information provided and displayed in the application was sufficient. Most participants explained that with all the information available on the screen, the holy visit would

be more easy and relaxing regarding not being lost or forgetting to perform any of the duties. This aspect of the design was achieved by following the visibility of system status heuristic of Nielsen (1994), which emphasises the fact that the system must always keep the user updated with what is happening. Also, this was indicated through the Symbian UIQ by Jones and Marsden (2006).

As a result of the well-structured nature of the application, most participants were pleased with it. This was due to the fact that the idea is new and unique in Jordan. In addition, the application is easy to use and to interact with. They would benefit from the application by being lost in Makkah or Madina in addition to not forgetting any part of the pilgrim journey. 
For the cultural aspect, Jordan is a masculine community and it applies high power distance. Both males and females were uncertain whether they found it easy or not. It was explained that this was the first time that the participants dealt with such technology, and they were afraid that something wrong would happen if they misused the application which as a consequencecould possibly end up with being lost or performing the worship in an inaccurate way.

These observations link directly to Trompenaar's(1993) dimension of individualism versus communitarianism. The Jordanian community is communitarian. This community relies on the fact that a person reports back to the group about an issue and then the group approves the final decision about the issue. Communitarians believe and practice that they have the ability to achieve more if they stick together as a group. This is typical of the way Jordanian people behave when it comes to the decision making process.

If they are to refuse or agree on something they will certainly acts toward it as a group. That is why both females and males nearly have no differences in opinion in such communities.

The participants in the study found the application useful, and they felt it was their duty and moral obligation to let other members of the community to know about it. This is a typical collectivist attitude. Also (participants of the study) felt that they would achieve more goals advise their pares in the community of anything might give them more benefit in their life.this is typical communitarianism.

They also added that the design is quite well thought out and they would recommend it to the others because they did not have any difficulties using it, regardless of their experience of using mobile handset/internet technology.

\section{Conclusion}

From this study, it has shown that there is significant acceptance in the uptake process of Hajj and Umra mobile application in Jordan. This was due to the successful understanding of the culture and its dimensions. These results could be easily generalized on any Muslim Cultures and nations due to its ease of use and flexibility.

\section{Aknowledgment}

"This research is funded by the Deanship of Research and Graduate Studies in Zarqa University /Jordan"

\section{REFERENCES}

[1] BEATTY, P., REAY, I., DICK, S. and MILLER, J., 2011. Consumer trust in e-commerce web sites: a metastudy. ACM Computing Surveys (CSUR), 43(3), pp. 14.

[2] BOND, M.H. and HOFSTEDE, G., 1989. The cash value of Confucian values. Human Systems Management, 8(3), pp. 195-200.

[3] BUChANAN, G., FARRANT, S., JONES, M., THIMBLEBY, H., MARSDEN, G. and PAZZANI, M., 2001. Improving mobile internet usability, Proceedings of the 10th international conference on World Wide Web 2001, ACM, pp. 673-680.

[4] Buchanan, G., Farrant, S., Jones, M., Thimblepy H., Marsden, G., Pazzani, M. 2001.Improving Mobile Web Usability.In Proceedings of the $10^{\text {th }}$ international conference on world widewep (Hong Kong, Hong Kong, May 01-05, 2001).WWW'01. ACM Press, New York, NY, 673-680

[5] CARROLL, J.M. and ROSSON, M.B., 2008. Theorizing mobility in community networks. International Journal of Human-Computer Studies, 66(12), pp. 944-962.

[6] CARROLL, J.M., 2000. Making use: scenario-based design of human-computer interactions. The MIT press.

[7] CDATA-LUHMANN, N., 1979. Trust and Power.

[8] Choi B, Lee I, Kim J, Yunsuk J 2005 A qualitative cross national study of cultural influences on mobile data service design. Conference on human factors in computing systems, CHI 2005. ACM Press, New York, pp 661-670

[9] Computing Systems, San Jose, USA, 2007, pp. 23452350.

[10] Coolican, H. 2004 Research Methods and Statistics in Psychology .4th ed. London: Hodder\& Stoughton.

[11] COOLICAN, H., 1994. Research methods and statistics in psychology. Third Edition edn. Hodder Education.

[12] COOLICAN, H., 2004. Research methods and statistics in psychology. Fourth Edition edn. Hodder Education.

[13] Coursaris, C.K., Kim, D.J., 2007. A research agenda for mobile usability. In: Proceedings of the ACM $\mathrm{CHI} 2007$ Conference on Human Factors in 
[14] Dix, A., Finlay, J., Abowd, G., and Beale, R., 2004, Human-Computer Interaction, $3^{\text {rd }}$ edition (Prentice Hall).

[15] Evelyn P. Rozanski and Anne R. Haake. 2003. The many facets of $\mathrm{HCl}$. In Proceedings of the 4th conference on Information technology curriculum (CITC4 '03). ACM, New York, NY, USA, 180-185.

[16] Hofstede,G. 2001.Culture's Consequences: comparing values, behaviors, institutions, and organizations across nations ( $2^{\text {nd }}$ Ed.). Thousand Oaks, CA: SAGE Publications

[17] Hornb_k, K., 2006. Current practice in measuring usability: challenges to usability study and research. International Journal of Human Computer Studies 64 (2), 79-102.

[18] Howart, J., Smith-Jackson, T, Hartson, R. 2009.Supporting novice usability practitioners with usability engineering tools. International journal of human computer studies, Vol 67, issue 6 . Pp 533-549.

[19] HOWARTH, J., SMITH-JACKSON, T. and HARTSON, R., 2009. Supporting novice usability practitioners with usability engineering tools. International Journal of Human-Computer Studies, 67(6), pp. 533-549.

[20] Jones M, Marsden G. 2006 Mobile Interaction Design.UK :John Wiley \&sons,

[21] Kaikkonen, A. 2008 Full or tailored mobile web - where and how do people browse on their mobiles? Proceedings of the international Conference on Mobile Technology, Applications, and Systems (Yilan, Taiwan, September 10-12, 2008). Mobility 08, pp1

[22] Lee, K. B., Grice, R.A., 2004. Developing a new usability testing method for mobile devices. In: Proceedings of the International Professional Communication Conference, Piscataway, USA, 2004, pp. 115-127.

[23] Ling, C., Hwang, W., Salvendy, G., 2006a. A survey of what customers want in a cell phone design. Behaviour\& Information Technology 26 (2), 149-163.

[24] Love, S., 2005. Understanding Mobile Human- Computer Interaction .Oxford: Elsevier Ltd.

[25] MOORE, G.C. and BENBASAT, I., 1991. Development of an instrument to measure the perceptions of adopting an information technology innovation. Information systems research, 2(3), pp. 192-222.

[26] NIELSEN, J., 1994. Enhancing the explanatory power of usability heuristics, Proceedings of the SIGCHI conference on Human factors in computing systems: celebrating interdependence 1994, ACM, pp. 152-158.

[27] Nielsen, J., 1993. Usability Engineering. Oxford: Academic Press. NJ.

[28] Norman, D. A. \& Draper, S. W. 1986 User-Centered System Design: New Perspectives on HumanComputer Interaction., Hillsdale: Lawrence Earlbaum Associates

[29] ROSE, G.M., EVARISTO, R. and STRAUB, D., 2003. Culture and consumer responses to Web download time: A four-continent study of mono and polychronism. Engineering Management, IEEE Transactions on, $50(1)$, pp. $31-44$.

[30] SALAM, A., IYER, L., PALVIA, P. and SINGH, R., 2005. Trust in e-commerce. Communications of the ACM, 48(2), pp. 72-77.

[31] SALAMON, S.D. and ROBINSON, S.L., 2008. Trust that binds: The impact of collective felt trust on organizational performance. Journal of Applied Psychology; Journal of Applied Psychology, 93(3), pp. 593.

[32] SCHADEWITZ, N., 2009. Design patterns for cross-cultural collaboration. International Journal of Design, 3(3), pp. 37-53.

[33] Venkatesh, V., M. Morris, G. Davis, F. Davis. 2003. User acceptance of information technology : Toward a unified view. MIS Quart Vol 27 No. 3/September 2003.

[34] Weiss, S., 2002. Handheld Usability.Chichester: John Wiley \& Sons

\section{Author' biography with Photo}

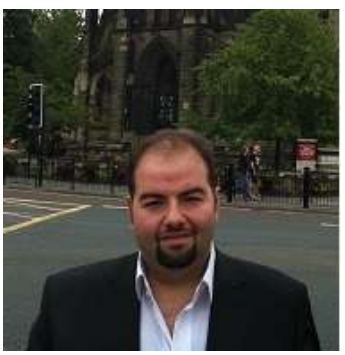

Dr. Firas Omar is an assistant professor in the software engineering departmet at Zarqa University, Jordan. He recived his Ph.D in information systems from Brunel University, London, UK, 2012. He received his MSc degree in Network systems from Sunderland University, Sundeland, UK 2006 and his BSc degree in computer science from Applied Science University, 2004. His research interest mainly conserned in $\mathrm{HCl}$ beside the cultural and usability studies. In addition, he is interested in the studies of trust and mobility. 


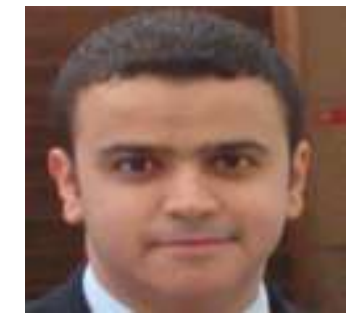

Dr. Tareq Alhmiedat: is a professor assistant in Computer Science Department at Zarqa University. He received a Ph.D. in Computer Science from Loughborough University, Loughborough, UK, 2009. MSc in Software Engineering from the University of the West of England, Bristol, UK, 2006, and a BSc degree from Applied Science University, 2004. His research interests including tracking mobile targets through Wireless Sensor Networks, Robotic Systems, and Home automation and remote control application.

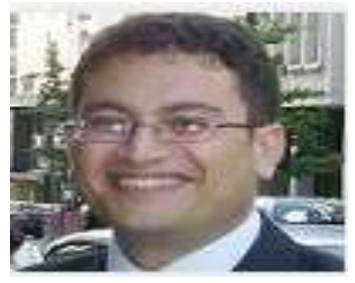

Dr.Mohammad Hassouna is a Lecturer in Enterprise and Mobile Applications Development at the university of Greenwich, UK. His research interests focuses on customer intelligence, customer satisfaction and customer retention. My main role in this position is to develop strategic decision support systems for CRM applications by employing a range of tools and techniques including: modelling simulation, data mining,artificial intelligence and advanced statistical analysis. 